\title{
NUCLÉAIRE, UNE (DÉ)RAISON D’ÉTAT
}

\section{Entretien avec Bernard Laponche}

\section{Entretien réalisé par Barnabé Binctin, Simon Cottin-Marx}

\author{
La Découverte | « Mouvements »
}

2018/3 n 95 | pages 165 à 184

ISSN 1291-6412

ISBN 9782348037511

DOI 10.3917/mouv.095.0165

\section{Article disponible en ligne à l'adresse :}

https://www.cairn.info/revue-mouvements-2018-3-page-165.htm

Distribution électronique Cairn.info pour La Découverte.

(C) La Découverte. Tous droits réservés pour tous pays.

La reproduction ou représentation de cet article, notamment par photocopie, n'est autorisée que dans les limites des conditions générales d'utilisation du site ou, le cas échéant, des conditions générales de la licence souscrite par votre établissement. Toute autre reproduction ou représentation, en tout ou partie, sous quelque forme et de quelque manière que ce soit, est interdite sauf accord préalable et écrit de l'éditeur, en dehors des cas prévus par la législation en vigueur en France. Il est précisé que son stockage dans une base de données est également interdit. 


\section{ITINÉRAIRE}

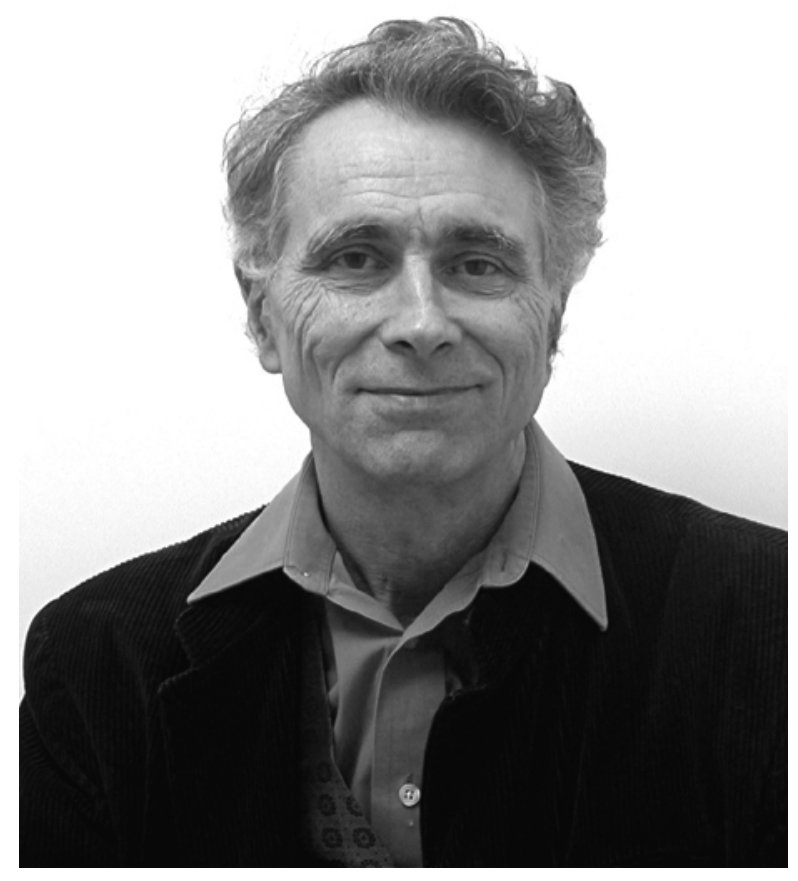




\section{Nucléaire, une (dé)raison d'État Entretien avec Bernard Laponche}

\author{
ENTRETIEn RÉALISÉ \\ PAR BARNABÉ \\ Binctin et Simon \\ COTTIN-MARX *
}

Depuis une cinquantaine d'années, Bernard Laponche nous alerte sur les dangers de l'atome. Dans cet itinéraire, ce physicien nucléaire qui fut membre du Commissariat à l'Énergie Atomique (CEA), nous raconte comment son expérience syndicale l'a amené à découvrir le risque nucléaire, pour les travailleurs de cette industrie et plus largement pour la population. A travers son parcours militant et professionnel passant par la CFDT, Global Chance, ou le cabinet du ministère de l'Environnement, il nous révèle la folie d'un État français enferré dans une politique pronucléaire. Cet ancien directeur de l'AFME (I'ancêtre de l'Agence de l'environnement et de la maîtrise de l'énergie - ADEME) a aussi imaginé et construit des alternatives : il est un infatigable défenseur des économies d'énergie et des énergies renouvelables.

\footnotetext{
* Physicien nucléaire.

** Membres du comité de rédaction de Mouvements.
} 
mon père - mais l'objectif Normale Sup était beaucoup trop difficile pour moi. Les gens ne se rendent pas compte, parce que Polytechnique a une renommée très élevée également, mais cela n'a rien à voir : le concours d'entrée à Normale Sup est bien plus difficile que celui de Polytechnique. Au premier, par an, il y avait trente personnes reçues, contre trois cents au deuxième.

Je ne savais pas vraiment ce qu'était Polytechnique, même si j'avais compris qu'il y avait une affaire militaire dans l'histoire puisque j'avais passé le Conseil de révision pour savoir si j'étais apte : une sorte d'examen de santé physique. Comme j'étais grand et très maigre, je n'aurais même pas dû être reçu. Je suis donc rentré à l'école Polytechnique où l'on devient militaire de carrière. C'est tout à fait étonnant, et même anormal : on rentre dans une école militaire qui fonctionne comme une caserne, dirigée par un général, dans laquelle vous passez deux ans avec des règles et un fonctionnement militaires. À l'époque, c'était encore dans Paris, à la montagne Sainte-Geneviève, donc plus agréable que maintenant, où le plateau d'Orsay est absolument lugubre. C'était une vraie vie de caserne, avec les deux promotions de trois cents élèves chacune. Chaque promotion a une couleur, rouge ou jaune, et pour moi ce fut la jaune. Tout le monde est pensionnaire, dans des chambres à huit lits et à côté, une salle avec huit petits bureaux et huit petites armoires. Le lieu de travail est extrêmement confiné. Très rapidement, on nous apprend le maniement des armes, il y avait des heures de sport et des heures innombrables pour apprendre à défiler aux cérémonies du 14 juillet.

\section{M. : N'avez-vous pas aussi appris à être ingénieur à Polytechnique?}

B. L. : Très peu. Cette atmosphère m'a complètement déstabilisé. C'était très difficile à vivre, vraiment. On était régulièrement puni parce qu'on avait oublié sa cravate ou parce que les lacets de souliers étaient mal ficelés. Il y avait une atmosphère disciplinaire, et ça compte parce que les points de discipline étaient intégrés dans le classement. Donc si vous vouliez une bonne place à la sortie de Polytechnique, il fallait être sage.

\section{M. : La discipline militaire primait-elle trop sur l'enseignement de l'in- génierie?}

B. L. : On ne peut pas dire ça. Mais cela m'a marqué, parce que je pensais que ce serait des études où l'on apprendrait beaucoup de choses. À l'inverse, je suis tombé sur cette atmosphère assez pénible et du coup, j'ai eu beaucoup de mal à y travailler, comme beaucoup d'autres élèves d'ailleurs. Je suis rentré au rang $150^{\mathrm{e}}$ et je suis sorti $150^{\mathrm{e}}$, à peu près, en ayant mal travaillé. Les suivants ne devaient pas avoir beaucoup travaillé non plus. Je considère que d'un point de vue scolaire, le niveau était assez lamentable. Ce sont des cours de maths, de physique, de chimie, de mécanique, avec des professeurs assez âgés et souvent difficiles à comprendre, sans aucune matière du type philosophie ou histoire. Seul le professeur Leprince-Ringuet m'a paru intéressant, c'est pour cela que je me suis tourné vers la physique alors que j'étais plutôt matheux de goût 
et de formation. À la fin de ces deux années, il y en avait deux autres de service militaire à l'époque, et je les ai faites dans la marine. C'était plutôt sympathique, et en parallèle, j'ai passé une licence de mathématiques, car je voulais rattraper le temps perdu, en quelque sorte.

\section{M. : C'est aussi une période trouble pour la France, qui est en guerre avec ses colonies : y avez-vous participé?}

B. L. : Non. Grâce à la marine, j’y ai échappé et je n'ai pas été mêlé directement à la guerre d'Algérie. Quand on sort de Polytechnique, on est officier d'active. Moi, j'étais lieutenant de vaisseau de deuxième classe.

Quand on est parmi les premiers, on sort dans les corps : corps des mines, corps des ponts, corps des télécommunications, corps des poudres, etc. et on va dans une école d'application (l'école des Mines, l'école des Ponts, etc.) en restant dans la haute administration. Cela va jusqu'au $130^{\mathrm{e}}$, le dernier corps étant je crois l'armement. Il y a une espèce de tradition qui fait que votre voie est tracée : vous êtes, dès votre jeune vingtaine, dans l'élite de la haute administration française, poste assuré jusqu'à soixante-cinq ans. Un certain nombre passent toutefois dans le privé après quelques années dans l'administration. Au-delà de 130, pour rester au service de l'État, il faut être militaire. Et toute cette formation est censée y préparer. Mais en fait, personne ne choisit cela : dans ma promotion il n'y en a eu que quatre ou cinq, je crois. Autrement dit, il faut démissionner et chercher un travail. Moi je ne savais pas très bien ce que je voulais faire après tout ça, je savais seulement que j'avais envie de travailler dans l'international. En discutant avec le professeur Leprince-Ringuet, j'avais imaginé l'existence d'une activité (qui n'existait pas à l'époque) d'attaché scientifique dans les ambassades, poste qui a été créé par la suite d'ailleurs. Donc je suis allé voir le ministère des Affaires étrangères, où l'on a trouvé l'idée intéressante. Pour pouvoir assurer un tel poste, on m'a conseillé de passer quelque temps en un lieu où se pratiquait la science française. Le top du top à cette époque-là, d'après Leprince-Ringuet, c'était la science des réacteurs nucléaires à Saclay, principal centre du Commissariat à l'énergie atomique, le CEA, fondé en 1945. C'est comme ça que je m’y suis retrouvé embauché, au Service de physique mathématique de la Direction des piles atomiques, dont le patron était Jules Horowitz. Pendant douze ans, j’y ai travaillé sur la physique des réacteurs nucléaires. Je me suis spécialisé sur ce sujet, notamment en suivant le troisième cycle créé, l'année où je suis arrivé, par Jules Horowitz. C'était un homme tout à fait remarquable, pour qui j'avais beaucoup de respect et qui dirigeait toute la physique des réacteurs en construction et en projet à l'époque (les réacteurs à uranium naturel graphite gaz, première génération du programme français de centrales nucléaires). On est en 1961, et il y a seulement les petits réacteurs de Marcoule, Brennilis n'est pas encore construit. Et je n'ai jamais donné suite à l'idée d'attaché scientifique, ensuite.

J'ai donc travaillé sur les premiers réacteurs d'EDF : Chinon 1, Chinon 2, Chinon 3. Dans ce Service de physique mathématique, j'ai fait les calculs sur les réacteurs nucléaires et une thèse d'État qui a fini par aboutir en 
1972, sur l'interprétation d'expériences pour déterminer les propriétés neutroniques du plutonium - très théorique, avec des équations partout. J'ai bien travaillé, c'était intéressant. J'ai enseigné un peu : la physique des réacteurs et le génie atomique. Et je me suis fait propagandiste, on peut le dire, du nucléaire : cela consistait à faire des cours et des conférences qui expliquaient comment tout cela marchait. Moi, cela me satisfaisait tout à fait, dans ce contexte où la France voyait fleurir ses premières centrales nucléaires, dans les années 1960.

\section{- Mai 68 et le début de l'antinucléarisme}

\section{M. : Qu'est-ce qui vous a fait passer de propagandiste du nucléaire, comme vous vous qualifiez, à militant anti-nucléaire?}

B. L. : Le virage, c'est mai 68. Jusque-là, j'étais syndiqué à la CFDT, mais syndicaliste passif. J'étais syndiqué par principe : je considérais que, quand on arrivait dans une boîte, on se syndiquait. En 1968, le mouvement est très puissant à Saclay. Tout est en grève au CEA. Il y a des élections de conseils partout, à tous les échelons : conseil de service, de département, et conseil de direction à Saclay. Et Saclay, à cette époquelà, c'était quand même une petite ville de 8000 personnes qui travaillaient sur les réacteurs nucléaires, la physique fondamentale, des activités très diverses... J'ai été élu aux conseils des différents niveaux et pendant toute cette période je me suis retrouvé secrétaire du comité d'action, qui est devenu ensuite un comité intersyndical d'action, présidé par Bernard Boudouresque, polytechnicien et prêtre-ingénieur, très apprécié notamment pour ses positions contre l'arme nucléaire et qui est devenu un ami. Donc, je fus très impliqué dans toute cette période de mai 68 .

Mai 68, c'était des questions à caractère social, les droits des travailleurs, la différence entre les cadres et les non-cadres, des tas de considérations de caractère social et anti-hiérarchique. Le mouvement était très massif : c'était assez considérable. Mais il n'a pas du tout été question de nucléaire, cela n'a pas été un sujet chez nous. À cette époque, cette question était peu relayée en France. Les seules discussions sur le sujet portaient sur le nucléaire militaire, car beaucoup de scientifiques du CEA étaient contre - à la suite de Joliot-Curie en particulier. En 1968, à la suite de discussions avec la direction, on a obtenu toute une série de droits supplémentaires comme la création de sections syndicales dans les centres militaires - ça, c'était très important - et puis les conseils d'unité comme on les appelait, que j'avais beaucoup défendus, qui consistaient à promouvoir l'idée que les gens pouvaient élire un conseil de représentants du personnel pour dialoguer avec la direction. C'est apparu dans la convention de travail du CEA, je pense que ça existe toujours d'ailleurs, et c'était assez nouveau d'avoir ces conseils élus dans un système très hiérarchique.

\section{M. : Est-ce qu'il y a eu de la résistance de la part de la direction?}

B. L. : Les négociations ont duré presque un an, entre les syndicats et la direction, et on a obtenu pas mal de choses. Il y a eu la grève générale 
du mois de Mai, et puis après, en septembre, une manifestation dans Saclay. Moi, pendant le mois de Mai je n'ai quasiment pas bougé, j'ai été deux fois à la Sorbonne pour voir, mais vraiment on restait sur le Centre, on était en grève, certains travaillaient, d'autres pas, et c'était le Comité d'action qui organisait les choses. C'était très particulier. Mai 68 reste un grand événement.

Les choses, comme partout, sont retombées : dans un premier temps, il y avait les assemblées générales que l'on faisait tous les jeudi à 13h, on avait obtenu dans les discussions d'avoir le jeudi après-midi en temps libre pour qui voulait faire la révolution, jusqu'à la signature de la Convention de travail. Au début il y avait plein de monde en AG, on était 400 à 500 dans le très grand amphithéâtre - et moi à chaque fois j'étais l'animateur de ce moment-là - et puis petit à petit, ça a réduit et je ne sais pas combien on était à la dernière, mais pas très nombreux. On a quand même tenu jusqu'au bout, à peu près six mois à partir d'octobre 1968. Et c'est un phénomène très intéressant, parce qu'on l'a retrouvé à d'autres époques et sur d'autres sujets, il y a une espèce d'emballement à un moment et puis ça retombe... L'enthousiasme a été cassé en juin 1968 par les élections législatives, qui ont été un raz-de-marée de la droite. Mais toute cette époque a été très importante pour moi.

\section{M. : Est-ce un virage personnel?}

B. L. : Oui, je suis devenu militant. Jusqu'à trente ans, je ne l'étais pas, c'est à partir de Mai 68 que je le suis devenu.

\section{M. : À quel moment se sont posés les problèmes du nucléaire?}

B. L. : Les interrogations sur le nucléaire sont arrivées en partie avec le changement de technologie : la fin du graphite gaz et le choix des réacteurs à eau ordinaire. Ce choix a entraîné une crise sociale au CEA : il y a eu, non pas des licenciements, mais des primes de départ, surtout dans les usines et à Marcoule notamment. Il y a donc déjà eu une crise sociale liée au choix de la politique nucléaire.

Pour moi, c'est la découverte des risques du nucléaire pour les travailleurs et les populations.

\section{- La naissance du risque nucléaire}

\section{M. : En quoi ce changement de technologie entraîne-t-il un risque sup- plémentaire?}

B. L. : La technologie graphite-gaz était française, très liée au CEA, et là on passait à une technologie Westinghouse, donc américaine. Cela avait des conséquences sur les activités du CEA. La question des risques pour les travailleurs devenait aussi de plus en plus visible.

En tant que militant à la CFDT, je fus délégué du personnel pendant deux ans. Par mes activités syndicales, j'ai côtoyé les gens de La Hague et de Marcoule, les ingénieurs de radioprotection, bref tout l'éventail du nucléaire. Avec ce changement de technologie, on a commencé à s'inté- 
resser à ce qu'était le nucléaire, à quoi il servait, etc. Parce que jusquelà, chacun faisait son travail sans connaître celui des autres, ni l'ensemble des problèmes de cette technique très particulière. Ce qui est toujours le cas : le nucléaire est une activité très compartimentée. "L'énergie, c'est quoi ?", "pourquoi produit-on?", " est-ce que le nucléaire est dangereux? ": toutes ces questions n'étaient pas du tout à l'ordre du jour. Ce sont les conditions de travail qui m'ont fait comprendre qu'il y avait des risques. Et que les ouvriers de Marcoule et de La Hague étaient au contact de cette matière nocive. Ce n'était alors connu que par quelques personnes qui suivaient les dossiers, comme Jean-Claude Zerbib, qui était ingénieur de radioprotection et responsable CFDT : lui, il avait une très bonne connaissance de ces risques. Il savait, en tant que délégué du personnel, qu'il y avait des maladies qui n'étaient pas reconnues.

\section{M. : C'est à partir de ce moment-là que naît le débat autour du nucléaire?}

B. L. : C'est un mouvement général. Dans la société des années 1960, il $\mathrm{y}$ avait très peu de personnes qui critiquaient le nucléaire civil. Puis, il y a eu des associations, notamment Survivre et Vivre dans laquelle militait Alexandre Grothendieck, qui ont commencé à en faire un vrai sujet, en particulier sur la question des déchets et de leur radioactivité. À Saclay, la section syndicale CFDT a organisé une assemblée générale (AG) à laquelle Grothendieck fut invité, au début des années 1970. La salle était pleine. Grothendieck a fait un discours expliquant que les déchets, c'était dangereux, qu'il y en avait à côté de Saclay qui étaient mal gérés, qu'il y avait des fuites radioactives... Un discours très alarmiste, et très antinucléaire. La hiérarchie était venue - parce que c'était M. Grothendieck, un grand mathématicien - et ils étaient fous de rage. C'était la première fois qu'il y avait, in situ, dans le temple du nucléaire, une AG avec un scientifique très respecté qui expliquait à tous ces braves gens que ce qu'ils faisaient était dangereux. Il a aussi fait le lien avec le nucléaire militaire, même si d'autres lui ont répondu qu'ils ne faisaient pas du militaire, mais de la recherche fondamentale. Mais à Saclay, il y avait bien des gens qui s'occupaient des sous-marins nucléaires par exemple, donc il y avait une ambiguiité au CEA.

Cette AG a fait scandale. Je me souviens que j'avais été interrogé par des journalistes et notamment par un quotidien de courses de chevaux - Turf, je crois - qui m'a interviewé sur ces histoires de déchets radioactifs : je me suis retrouvé en une, avec une photo et un titre qui disait "Un savant du nucléaire nous explique que les déchets radioactifs sont dangereux ". Les amateurs de pari équestre ont dû être surpris, mais c'était assez rigolo. Heureusement que j'étais syndicaliste et donc protégé. Cela a beaucoup joué : le fait de représenter le syndicat vous autorise une beaucoup plus grande liberté d'expression, cela offre une protection beaucoup plus importante - souvent les gens ne comprennent pas cela.

Après 1968, je me suis retrouvé au Bureau national du syndicat et avec Jean-Claude Zerbib, on s'est mis à travailler sur des brochures à destination des adhérents de la CFDT : sur la technique des réacteurs (comment 
1. Cet accident nucléaire s'est produit le 28 mars 1979 dans la centrale nucléaire de Three Mile Island, en Pennsylvanie (États-Unis). ça marche), le combustible (l'uranium, le retraitement), la sûreté nucléaire et les accidents, la radioactivité et la radioprotection, les conditions de travail... Avant, il n'existait rien, aucun document sur le sujet. Ces documents ont été très appréciés, y compris par la direction du CEA, et très vite, $L e$ Seuil, en la personne de Jean-Marc Levy-Leblond, nous a proposé d'éditer un livre rassemblant et complétant toutes ces brochures. Ce fut L'électronucléaire en France, signé par le syndicat CFDT de l'énergie atomique et édité en 1975. Beaucoup de gens s'en servent encore aujourd'hui - il a été complété et réédité en 1980 sous le titre Le dossier électronucléaire.

C'était une grande première, et tous les directeurs avaient ce livre pour chercher un renseignement. Moi j'avais fait la partie technique et la partie sûreté nucléaire. Et j'en ai conclu que c'était trop dangereux. C'est l'écriture et la réalisation de cet ouvrage qui m'ont fait changer d'avis.

\section{M. : Personne ne prenait alors en compte la mesure du risque, au CEA ?}

B. L. : Comme tous les gens du nucléaire, on misait sur la probabilité extrêmement faible d'un accident grave... Il y avait une certaine connaissance du risque, mais on considérait que toutes les précautions étaient prises, que les réacteurs étaient très bien construits, qu'on trouverait une solution pour les déchets, etc. La responsabilité de la sûreté était assurée à ce moment-là par le CEA lui-même.

\section{M. : Un risque considéré donc comme acceptable?}

B.L. : Oui, comme aujourd'hui d'ailleurs. Les 58 réacteurs français ont été conçus sur l'hypothèse que l'accident grave n'était pas possible. L'expérience a prouvé le contraire avec Three Mile Island ${ }^{1}$ en 1979. Mais c'était la conviction générale pour toutes les centrales. Moi-même, je n'avais jamais vraiment regardé ce problème et donc j'avais la même conviction. Comment vous dire, c'était une sorte de croyance...

\section{- L’influence du syndicalisme}

\section{M. : C'est ce travail mené avec la CFDT qui ébranle en profondeur la doctrine officielle?}

B. L. : Il y a eu aussi les travaux d'autres scientifiques français, regroupés dans le GSIEN (Groupe des Scientifiques pour l'Information sur l'Énergie Nucléaire) fondé en 1975 par Monique et Raymond Sené - leur publication, La Gazette nucléaire, en est aujourd'hui à son numéro 286. Plus tout ce qui se faisait à l'époque aux États-Unis, également. Mais c'est quand même de la CFDT qu'est partie une première contestation du nucléaire civil, à l'intérieur même de l'institution qui en était le premier promoteur.

À la fin des années 1960 et au début des années 1970, on voit arriver le mouvement antinucléaire, qui est lié aux mouvements de 68 et à cette remise en cause générale. À cette époque, la CFDT sort un autre livre qui s'appelle Les dégâts $d u$ progrès, qui procède de la même réflexion. Il y a le Club de Rome, aussi. Il y a toute une ambiance post-soixante-huitarde, avec beaucoup de ramifications, qui commence à mettre en doute 
la science, la croissance, etc. Et nous, on s'inscrit parfaitement dans cette démarche. Et d'ailleurs, dans Les dégâts du progrès, il y a un chapitre sur le nucléaire qui a été écrit par le secrétaire général du syndicat de la CFDT au CEA de l'époque, Jean-François Mougel.

À la même époque, il commence aussi à y avoir des luttes à La Hague sur les conditions de travail, mais aussi sur le fait que le CEA veut en faire une société à part dans l'objectif d'une privatisation. En décembre 1976 se tiennent les assises du nucléaire à La Hague, avec un monde fou, des stands, des conférences, une grève générale, etc. C'est à ce moment-là qu'on fait un film, intitulé Condamnés à réussir et réalisé par François Jacquemain. C'est un film remarquable, joué par les militants de la CFDT de La Hague eux-mêmes qui ont reconstitué leur lieu de travail - tellement bien que tout le monde a cru qu'on avait tourné dans l'usine. Le film raconte leur quotidien : c'est une usine chimique où il y a des tuyaux partout, qui transportent une matière extrêmement dangereuse, y compris du plutonium puisque le but de cette usine est d'extraire ce plutonium. Les travailleurs de La Hague interviennent pour faire des mesures, changer certains équipements ou décontaminer des locaux. Ils sont en scaphandre et ils descendent dans l'usine, pour changer une pompe par exemple. Il faut vidanger, sectionner des tuyaux, faire des soudures, remplacer des équipements et tout ça en milieu radioactif - pas tant avec un risque de rayonnement, mais plutôt de contamination : si vous prenez un micrograin de plutonium, vous êtes à peu près sûr d'avoir un cancer. Et si en sciant, vous coupez votre gant... Le film explique ces conditions de travail, très difficiles.

\section{M. : Combien de réacteurs y avait-il, à l'époque?}

B. L. : Six réacteurs de puissance "graphite-gaz " dans les centrales d'EDF, mais aucun de la nouvelle génération, à eau ordinaire. C'est le début de la construction de Fessenheim, par exemple, qui démarre en 1977. Le programme Messmer envisage alors de lancer six réacteurs par an, et la CFDT s'y oppose. Avec une double argumentation: sur les risques et sur la politique de l'énergie.

En 1973, je prends un virage radical : je lâche ma profession. J'arrête et je deviens permanent syndical au syndicat CFDT de l'énergie atomique. Il y avait le bureau du syndicat et on était trois permanents. Je suis resté trois ans permanent (1973-1975), et puis, pour des raisons personnelles qui n'avaient rien à voir avec le syndicat, j'ai arrêté : c'était un travail considérable, je passais ma vie à participer aux débats sur les sites, en campagne de contestation contre l'implantation des centrales nucléaires. J'ai voyagé à peu près partout en France.

\section{M. : Comment s'opère ce virage vers le syndicalisme, pourquoi l'enga- ger à ce moment-là, après douze ans de carrière au CEA : qu'est-ce qui vous décide?}

B. L. : Je ne pouvais pas continuer à travailler pour un projet que je considérais comme condamnable. Et ce n'est pas un choix si difficile, 
puisque comme j'étais permanent syndical, je restais employé par le CEA et je travaillais toujours à Saclay.

Fin 1975, j'arrête cette activité de permanent et la direction du CEA a été très correcte avec moi, elle m'a proposé un poste au Département des programmes, c'est-à-dire les études économiques générales. Là j'ai travaillé sur la prospective énergétique, ce qui m’intéressait énormément, car la question que je me posais à l'époque, c'était de savoir ce que l'on pouvait faire à la place du nucléaire.

J'ai alors présenté une thèse de troisième cycle en prospective énergétique. C'était intéressant parce que je travaillais à montrer qu'il pouvait y avoir des scénarios alternatifs et que les prévisions officielles de consommation d'électricité étaient très exagérées. Le CEA n'a pas voulu que je passe ma thèse en tant qu'agent du CEA, donc je l'ai passée à titre personnel à l'Université de Grenoble, avec Jean-Marie Martin comme professeur. Ils n'ont pas tenu compte de ce travail, évidemment, mais il a tout de même donné lieu à un rapport CEA, au plus haut niveau de publication.

Ce furent trois années très intéressantes, parce que j'ai changé de métier : j'étais toujours au CEA, mais plus du tout sur des questions de nucléaire, plutôt sur les politiques énergétiques. Cela m'a beaucoup formé. En parallèle, j'ai continué à avoir des activités critiques sur le nucléaire: je participais au groupe Énergie à la Confédération CFDT, et au bout de trois ans, la CFDT a obtenu que le CEA me détache à la Confédération. Je suis redevenu à nouveau permanent, pendant trois ans, en tant que secrétaire confédéral, moitié sur l'international avec Jacques Chérèque et moitié sur l'énergie, avec Michel Rolant, qui était le responsable du secteur économie-emploi. Je travaillais toujours sur les risques du nucléaire et les politiques alternatives. En 1979, on a fait une conférence de presse sur les fissures des cuves de Fessenheim - déjà.

\section{- Le tournant politique de 1981 et la création de l'Agence Française pour la Maîtrise de l'Énergie}

\section{M. : Comment évolue le monde politique, sur cette question?}

B. L. : Le PS, qui était plutôt pronucléaire, a également fait bouger ses lignes de son côté. Il y a eu un gros travail de Paul Quilès, en particulier. Les ONG étaient montées en puissance sur ces questions, notamment les Amis de la Terre. Donc cela donnait tout un mouvement - CFDT, ONG, PS - qui a abouti en 1979 à une pétition nationale qui demandait à revoir la politique énergétique. Elle était très critique par rapport au nucléaire et elle a été signée par tout le monde, François Mitterrand en tête. On était donc assez confiant avec les présidentielles qui arrivent. Tout se présente très bien, tous les candidats de gauche à la présidentielle - Mitterrand, Lalonde, Bouchardeau - prennent des engagements. Pourtant dès l'été 1981, on a compris que l'avenir était incertain. Les engagements pris ne sont pas respectés, sauf l'annulation du projet de centrale nucléaire à Plogoff. C'est devenu un symbole de la lutte antinucléaire, un peu comme le Larzac à l'époque et Notre-Dame-des-Landes aujourd'hui. C'était un 
des cas très populaires - il y a aussi eu Port-la-Nouvelle, dans le Sud. Plogoff, c'est un site particulier, où les Bretons ont bien bataillé et ils ont gagné, mais il est probable que si François Mitterrand n'avait pas été élu, la centrale aurait été construite... Pour le reste, l'autorisation d'agrandir la Hague a été donnée rapidement et en octobre 1981, Pierre Mauroy a prononcé un discours remarqué à l'Assemblée nationale, un discours complètement pronucléaire. On en est sorti en pleurs. En trois mois, tous les engagements de François Mitterrand sur le nucléaire avaient disparu.

\section{M. : Comment expliquez-vous ce retournement de situation?}

B. L. : C'est assez clair : EDF est une puissance extraordinaire en France, la CGT en est le premier syndicat, le PC est pronucléaire... le PS était donc partagé. Néanmoins, le Président de la République s'était engagé. Et c'était une première, puisque les précédents, Georges Pompidou et Valérie Giscard d'Estaing, avaient lancé le grand programme nucléaire. François Mitterrand, lui, dit qu'il veut revoir tout ça et s'engage à ne pas commander de nouvelles centrales, à réexaminer la politique énergétique. Mais en France, la haute administration est pronucléaire, la plupart des députés sont pronucléaires, et EDF est toute puissante. Ce qui est dramatique, c'est que cette situation n'a pas bougé.

Depuis les années 1970 j'ai participé, en tant que CFDT, à la Commission énergie du Plan. C'était quelque chose d'important à l'époque. Il y avait tous les patrons de l'énergie : d'EDF, des charbonnages, du gaz, du pétrole, etc. Plus les organisations syndicales. C'était vraiment un travail sérieux entre ces partenaires et l'État. Cette commission se réunissait, travaillait, puis concluait : on construit ça, on va faire ci, on va faire ça. Pendant les années 1970, l'orientation était pronucléaire, mais les avis contradictoires pouvaient s'exprimer.

En 1983, la Commission de l'énergie, dont le rapporteur était JeanMarie Martin, dit qu'il fallait arrêter de construire des centrales nucléaires, puisque du point de vue économique on n'en avait plus besoin... Mais on a continué à en construire et on s'est enfermé dans cette obstination, alors qu'en 1981 on aurait très bien pu réviser le programme, car il était basé sur des prévisions d'électricité absolument divagantes - comme je l'avais déjà dit dans mon travail de prospective. Paul Quilès a été écarté, alors qu'il aurait normalement dû prendre la responsabilité de l'énergie, et on s'est retrouvé comme des cons, on peut le dire. Complètement dépouillés. En contrepartie, François Mitterrand, qui ne voulait quand même pas perdre tous ses alliés, a créé en 1982 l'Agence Française pour la Maîtrise de l'Énergie (AFME), un organisme qui rassemblait l'Agence pour les économies d'énergie créée en 1974 et le Commissariat à l'énergie solaire, très dynamique dans la recherche. Il y avait des moyens importants, des moyens de recherche sur le renouvelable et l'efficacité énergétique qu'il n'y avait pas jusque-là. L'AFME était un instrument bien construit et Michel Rolant en a été nommé président. Il avait défendu tout ça dans la CFDT. C'était une nomination à la fois intelligente, car il connaissait bien ces sujets-là, et politiquement habile, puisque cela 
montrait à la CFDT que toutes ces bagarres n'avaient pas été inutiles, au moins sur la partie des alternatives. Et comme j'avais déjà travaillé avec Michel Rolant, en 1982 il m'a demandé de venir avec lui. Avec quelques autres d'ailleurs : il a eu la prudence de ne pas arriver tout seul dans cet organisme. J'ai pu mettre en application mon travail précédent. C'était une entreprise de 400 à 600 personnes. J'ai travaillé d'abord, au Département des programmes, puis je suis devenu Directeur général de 1984 à 1987, pendant trois ans.

Michel Rolant était soutenu par François Mitterrand qu'il connaissait bien. Ça ne se passait pas mal, même s'il y avait une hostilité d'EDF qui voyait ça évidemment d'un mauvais œil, et que la haute administration trouvait scandaleux que ça soit un syndicaliste qui en soit président, plutôt qu'un corps de mines, et de façon générale c'était anormal cette histoire. On a bien tenu, jusqu'à ce qu'en 1986 le gouvernement change. Le nouveau gouvernement est de droite et le ministre de l'Industrie et de l'Énergie est Alain Madelin. On a tenu un an. On a triomphé à la Conférence mondiale de l'énergie, en octobre 1986. L'AFME était partout. On avait un stand magnifique. C'était très glorieux. Et en 1987, la discussion budgétaire nous est défavorable, Michel et moi on avait accepté la réduction du budget : quelque chose de très fort, la moitié. On a dit " ok, on va travailler avec moins d'argent ", mais on a refusé de licencier. Parce que l'activité repose sur les gens et que la boîte marchait bien... Or le plan de l'État, du gouvernement, c'était de réduire les effectifs d'un tiers. Donc on a sauté là-dessus. Ils en ont profité : on arrivait en fin de mandat l'un et l'autre. Michel Rolant a été viré de façon scandaleuse : il devait y avoir un conseil d'administration où il comptait se défendre, et où il aurait peutêtre gagné, puisque sa position était de refuser les licenciements, et les syndicats l'auraient soutenu. Il y avait aussi des personnalités qui pensaient que détruire l'AFME c'était grave. Mais ils l'ont viré du conseil d'administration avant la tenue du CA...

\section{M. : À l'AFME, vous faisiez de la prospective, de la recherche, de l'indus- trie? Quelles étaient exactement ses missions?}

B. L. : C'était l'animation, la formation, la communication, la recherche, le conseil aux entreprises et aux collectivités territoriales, des aides financières aux collectivités locales et aux entreprises. Il y avait les services centraux qui suivaient les questions de recherche et de subvention, mais c'était décentralisé. Dans chaque région il y avait un Fonds régional de maîtrise de l'énergie où il y avait de l'argent de la Région et de l'AFME, pour soutenir les projets. Ça pouvait être des aides à l'industrie, au secteur tertiaire, à la communication auprès du grand public. C'était une Agence de promotion de la maîtrise de l'énergie, très décentralisée.

On a eu cinq milliards de francs d'investissement, pour essentiellement l'industrie et le grand tertiaire, pour faire de l'économie d'énergie. Ça s'appelait le Fonds Spécial de Grands Travaux (FSGT), qui distribuait des subventions jusqu'à $30 \%$ du coût du projet. Il y avait aussi une activité internationale assez importante, avec d'autres pays. En tant que directeur 
général, j'avais directement la responsabilité des services économique, international, communication et formation.

C'était très bien, et ça a été cassé. Ils nous ont virés tous les deux en juillet 1987, et est arrivé un Monsieur du corps de Mines, qui s'est institué président-directeur-général : il n'y avait plus qu'une seule tête (ce qui lui permettait aussi de cumuler les deux salaires). Il avait comme première mission de réduire les effectifs d'un tiers. Il l'a fait, mais par des primes de départ. Et ça n'a pas été les plus mauvais qui sont partis... dont des responsables. Sur les quatre chefs de service qui dépendaient de moi, trois sont partis. Les partants ont souvent créé ou rejoint un bureau d'études ou des associations afin de poursuivre la tâche, à l'extérieur. Il y a eu un déplacement de l'esprit de création vers la périphérie.

L'AFME a continué et est devenue l'ADEME (Agence De l'Environnement et de la Maîtrise de l'Énergie) en 1991: on lui a ajouté l'Agence pour la qualité de l'air et l'Agence pour les déchets.

De mon côté, je me retrouvais de nouveau au CEA, puisque j'en étais auparavant détaché. Le CEA était bien embêté de me récupérer. Ils m’ont proposé de travailler sur les applications militaires ou sur les déchets... enfin bon, on n'a pas eu des relations très cordiales. Je suis resté, j'ai pris des cours de dactylographie, mais pas suffisamment, car je tape toujours très mal, et de perfectionnement en anglais. J'ai fait ça trois mois, mais c'est devenu lassant. J'ai proposé de monter une équipe pour travailler à fabriquer des équipements performants d'électricité : réfrigérateur et autres. On m'a ri au nez, ce qui prouve qu'ils n'étaient pas très malins. Finalement, ça s'est terminé à l'amiable avec une prime de départ correcte. Ce qui m'a permis de créer en 1988 International Conseil Energie (ICE) avec Florence Rosenstiehl qui avait été cheffe du service de la communication à l'AFME. Un bureau d'étude dédié à l'efficacité énergétique et actif très rapidement à l'international - le nouveau patron de l'AFME avait demandé que l'agence ne passe pas de contrats avec nous. Assez vite, on a eu des contrats de la Commission européenne. On a travaillé dans les pays de l'Est et de la Méditerranée. ICE a grandi, c'était très intéressant. Du point de vue professionnel j'ai poursuivi dans ce domaine, avec une interruption qui m'a replongé dans le nucléaire : puisque je suis allé au cabinet de Dominique Voynet en 1998 et 1999. Et là j'ai été à 95 \% sur la sûreté nucléaire.

\section{- Faire face à l'État pronucléaire}

\section{M. : Quelle était votre mission exactement au ministère de l'Environne- ment et Aménagement du Territoire?}

B. L. : La sûreté nucléaire, c'était une direction générale qui avait deux tutelles : l'industrie et l'environnement. Pendant deux ans je m'en suis occupé, ainsi que de tout ce qui se passait dans le nucléaire.

Cela n'a fait que me conforter dans l'idée que c'est très dangereux. Et ça m'a confirmé que si le nucléaire est très dangereux, les dirigeants du nucléaire le sont aussi. C'est-à-dire qu'ils n'en avaient rien à cirer de 
ces questions-là : le risque d'accident et les déchets. Tous les présidents d'EDF, Edmond Alphandéry et François Roussely à l'époque, ou Jean-Bernard Lévy actuellement, c'est étonnant de voir à quel point ces questions, qui sont fondamentales, de sûreté et de déchets nucléaires, sont traitées lapidairement sous la forme : "Non, il n'y a pas de problème ".

M. : En 1981, Mitterrand a trahi. Mais là, en 1998-1999, vous êtes proche du pouvoir politique, vous y travaillez. Quelles marges de manœuvre avez-vous pour influencer la filière nucléaire?

B. L. : Très peu. Le succès principal, qui a été un succès parce que le truc était foireux, c'était Superphénix. Là, Lionel Jospin dit, "j'arrête Superphénix " et il a respecté sa parole. Il faut dire que ça ne fonctionnait pas, et la décision à prendre était de le redémarrer. Mais personne n'osait dire " on arrête " ou " on redémarre ". Lui au moins a dit " on arrête ". Il y a eu des tas de discussions après, il a fallu six mois de discussions pour qu'il soit véritablement arrêté, en février 1998, alors que son discours datait de juillet 1997. Plusieurs personnes étaient montées au créneau dire qu'il ne fallait pas l'arrêter. Le Parlement s'était ému. Mais Lionel Jospin a tenu bon. Aussi, il ne voulait pas perdre Dominique Voynet, car c'était important d'avoir des écologistes dans son gouvernement de gauche pluriel.

Elle a aussi obtenu de ne pas faire l'EPR (réacteur pressurisé européen), même si déjà ils le voulaient tous : CEA, EDF, Areva, et les autres ministres. C'était prêt. Mais Dominique Voynet a dit à Jospin, "si tu le fais je démissionne et je le fais savoir ". Comme il voulait la garder, comme il l'estimait en tant que ministre et politiquement, et comme il n'avait pas d'avis sur l'EPR, il a suivi son avis (l'EPR est ressorti avec Chirac qui a décidé de le lancer en 2005-2006).

Elle a aussi obtenu le fait de ne pas autoriser les demandes de rejets de la Hague. Mais autrement, c'était une guerre de tranchées permanente avec le ministère de l'Industrie qui était totalement pronucléaire, qui a soutenu à chaque fois Areva et EDF. Et elle a dû céder sur le laboratoire de Bure. Le gouvernement était majoritairement pronucléaire : il y avait Dominique Strauss-Kahn, Christian Pierret, Jean-Pierre Chevènement, Claude Allègre, et même Bernard Kouchner par moments. Dominique Voynet était très seule. Mais elle a permis d'avancer dans le domaine de la maîtrise de l'énergie et des renouvelables, Pierre Radanne devenant président de l'Ademe.

M. : Au-delà de ce ministère qui représente le lobby nucléaire, vous identifiez qui?

B. L. : Il n'y a pas de lobby nucléaire.

M. : Il y a tout de même des gens, des groupes porteurs de l'idéologie nucléaire, et qui mettent de côté le risque.

B. L. : Il n'y a pas de lobby nucléaire, ce n'est pas un lobby, car c'est à l'intérieur de l'État et pas à l'extérieur, comme un lobby du médicament qui fait en sorte que le parlementaire ou le ministre soit convaincu ou 
acheté. Pour le nucléaire, il y a un bloc solidaire au niveau de l'État, avec l'alliance indéfectible de la haute administration et des dirigeants des grands organismes qui sont, de fait, au sein de l'État: CEA, EDF, Areva... Cela commence maintenant à s'ébranler un peu, surtout en raison des questions économiques. Parce que tout ça est très cher, qu'Areva est quasiment en faillite et qu'EDF a des dettes énormes. Il y a des gens qui commencent à se dire que cette histoire n'est peut-être pas intéressante. Mais sur le nucléaire lui-même, indépendamment du côté économique, sur le danger qu'il représente, les risques, les déchets... il n'y a pas de questions.

M. : Dans votre parcours, il y a quelque chose dont vous n'avez pas parlé. Tchernobyl en 1986, qui commence à faire prendre conscience du risque. Puis Fukushima plus récemment.

B. L. : Le premier accident grave, ou en tout cas celui qui a ébranlé les certitudes sur le nucléaire c'est Three Mile Islande, mais ça a finalement été considéré comme pas si grave, parce qu'il n'y a pas eu beaucoup de radioactivité à l'extérieur. Un réacteur a été totalement détruit, mais bon, c'est tout. On entendait : "C'est des Américains, donc ce n'est pas très sérieux : le type n’a pas appuyé sur le bouton qu'il fallait, la pièce défaillante, ils auraient dû s'en apercevoir avant "... " C'est le secteur privé qui a fait n'importe quoi ". Il y avait un dessin de Konk dans le journal le Monde qui résume bien cet état d'esprit : un directeur d'EDF devant son bureau, et il déclare : "les ingénieurs américains sont des ânes "... Alors que tous les réacteurs français ont été construits sous licence américaine, sauf les quatre derniers qui ont d'ailleurs eu des problèmes.

L'accident de Tchernobyl a été très masqué en France par l'habituel " ce n'est pas dangereux ". Jean-Claude Zerbib² avait tout de suite repéré des niveaux de radioactivité anormaux, mais on lui a interdit d'en parler. Tchernobyl a été noyé dans le mensonge que la France avait été épargnée... Mais aussi par le fait que c'était un réacteur soviétique, que c'était " des nuls ", etc.

En France, le nucléaire est assimilé à l'hymne de La Marseillaise, au drapeau tricolore. Et donc on n'en discute pas. Cette situation a un peu changé depuis Fukushima, car on a eu la vision directe de l'accident, comme les tours du World Trade Center. On l'a vu !

Mais avant Fukushima, on ne peut pas dire qu'il y ait eu une réflexion au sein de l'État. Le mouvement antinucléaire, et ceux qui développaient un regard critique comme Monique Sené en 1975, n'arrivaient pas à toucher ce bloc, qui a toujours prétendu, et continue de prétendre que tout va bien.
2. Ingénieur de radioprotection du CEA.

\section{Alerter des dangers du nucléaire, promouvoir des alternatives passants par les économies d'énergie et les énergies renouvelables}

M. : Travailler pour une ministre écologiste, c'est un engagement. Vous en êtes où à ce moment-là, après le début des années 2000 ? 
B. L. : Toutes les années 1990, j’ai beaucoup travaillé avec ICE. J'étais souvent absent. J'étais beaucoup moins actif en tant que militant. Un petit groupe emmené par Benjamin Dessus et Martine Barrère a créé en 1992 l'association Global Chance et je les ai rejoints. Des scientifiques avaient lancé un appel pour un gouvernement de scientifiques : l'appel d'Heidelberg. Il y avait des gens très bien, mais nous n'étions pas d'accord. Nous étions un certain nombre à penser que la science doit être soumise aux discussions démocratiques, au contrôle citoyen. On est parti là-dessus en écrivant la charte de Global Chance : développer de l'expertise avec le public, avec les militants.

Cette association a produit deux numéros par an des Cahiers de Global Chance. C'était du bon travail et on est également intervenus comme experts critiques dans tous les débats nationaux sur l'énergie et sur le nucléaire. En 1995, le gouvernement avait confié à Jean-Pierre Soubiron, un ingénieur du Corps des mines, ancien directeur de l'industrie, la mission d'organiser un débat sur le nucléaire. Ce qu'il a fait de façon remarquable, avec des débats contradictoires. J'ai participé à un débat à Caen sur la sûreté des réacteurs des pays de l'Est. Il n'était pas du tout antinucléaire mais il disait " tout de même, il y a quelques problèmes... ". Son rapport a immédiatement disparu. Personne n'en a parlé, personne ne s'en souvient. Il a été ostracisé par ses camarades... On en est là ! Mais lui n'en avait rien à faire, il est passé dans le privé s'occuper d'autre chose.

Nous avons aussi participé au débat organisé par Nicole Fontaine, ministre de l'Industrie, en 2004. À celui sur l'EPR en 2005, les débats sur les déchets et sur Cigéo. Et on a poursuivi une activité de production d'études, d'articles, de tribunes, de livres, de conférences et de formations.

J'avais en parallèle, et surtout, mon métier sur l'efficacité énergétique à l'international. Dans les années 1990, j'ai travaillé avec l'équipe d'ICE en URSS, Russie, Roumanie, Ukraine, Palestine, etc. Puis, dans les années 2000, en Algérie, Tunisie, Chine, Nouvelle-Calédonie. Chaque fois je travaillais pour les États, sur les politiques d'efficacité énergétique du pays. Et aussi, en France, sur les premiers plans climat-énergie territoriaux, en Déodatie (Lorraine) ou régionaux, en Bretagne, Nord-Pas-de-Calais, Picardie, ainsi que sur des études pour la Commission européenne, le ministère français des Affaires étrangères, l'ADEME ou l'AFD.

\section{M. : Vous êtes encore très actif, vous avez participé à d'autres associa- tions, vous avez aussi eu un rôle de lanceur d'alerte.}

B. L. : J'ai continué à suivre en tant que Global Chance, les deux sujets : le nucléaire (la sûreté et les déchets) en écrivant des papiers, donnant des interviews. J'ai récemment participé au troisième Forum social mondial antinucléaire, qui s'est tenu à Paris début novembre 2017, et qui a été l'occasion de nombreuses rencontres internationales. On y a entendu des témoignages de travailleurs et de populations qui subissent les risques nucléaires, que ce soit dans les mines d'uranium ou dans celles qui ont connu des accidents majeurs, Mayak, Tchernobyl, Fukushima. 
Et puis de l'autre côté, j’ai continué à travailler sur les questions d'économie d'énergie, ce qui est ma spécialité, avec la participation à la création de l'association Énergie Partagée. Un mouvement qui vise à soutenir des projets citoyens, et de collectivités locales, qui s'est orienté essentiellement sur la production d'énergie renouvelable. Aussi, avec Benjamin Dessus, nous avons fait un gros travail sur le méthane comme gaz à effet de serre, en collaboration avec le climatologue Hervé Le Treut. J'essaye de susciter du travail sur les économies d'électricité. Car en France on gaspille l'énergie, notamment parce qu'on a trop de nucléaire et qu'il faut le consommer.

Je continue sur les deux voix de mes activités professionnelles, mais à titre militant, en liaison avec les ONG de ce secteur énergie, climat, nucléaire.

\section{- Le nucléaire, une (dé)raison d'État}

M. : Parlez-nous un peu justement de cette deuxième casquette, la politique de l'énergie. Comment voyez-vous le lien entre l'obstination nucléaire et les politiques énergétiques des trente dernières années?

B. L. : Les problèmes que l'on a aujourd'hui avec le nucléaire sont liés à des choix qui ont été faits dans les années 1970, avec des prévisions de consommation exagérément gonflées et sur lesquelles on s'est enferré. En France, au niveau des dirigeants, on fait de la prospective en "avenir certain ". Donc ce n'est pas de la prospective... Ils définissent un avenir certain, c'est-à-dire celui qui leur convient et il en découle la politique à suivre. C'est particulièrement dangereux, car, justement, l'avenir est incertain et que le secteur de l'énergie a connu des bouleversements, positifs ou négatifs, qu'il était bien difficile d'anticiper. Par exemple, EDF dit en 1975: "La consommation d'énergie en l'an 2000 sera de 1000 milliards de kWh ". Affirmation péremptoire : elle a été de 440 milliards. Le CEA disait : "Il y aura vingt Superphénix en France en 2000 ». Il y en a eu zéro. Et il n'est pas question d'étudier autre chose ! Au lieu de se rendre compte assez tôt que l'écart est clair entre ce qui a été prévu et la réalité, et de redéfinir une stratégie industrielle, ils s'obstinent, car ils pensent avoir raison.

À l'étranger, certains pays se sont aperçus de leurs erreurs. Quand l'Allemagne a décidé de sortir du nucléaire - pays par ailleurs vénéré sur les autres domaines -, sur l'énergie, les Allemands étaient "des imbéciles et des romantiques". Pour illustrer ce que je dis ; quand l'Allemagne a pris cette décision, il y a eu une séance à l'Assemblée nationale, qui était effrayante. On allait envoyer les chars. Sur vingt-trois intervenants, il y en a eu deux, Michèle Rivasi et Guy Hascoët, qui ont été prudemment approbateurs de la décision allemande, mais pour tous les autres c'était l'horreur. "Les Allemands ne nous ont pas consultés!" comme si nous, nous les avions consultés quand on a décidé de construire Fessenheim.

C'est devenu une maladie mentale. Ces gens ne s'interrogent pas. Jamais un pronucléaire ne m'a demandé mon avis, car ils auraient pu 
se dire qu'en tant que physicien, si Laponche pense ça, pourquoi ? Non, je suis devenu un grand malade. Et ça c'est très typique de la situation française. À l'étranger vous n'avez pas ça. En Allemagne, vous avez des instituts qui critiquent le nucléaire, en Angleterre, aux États-Unis, c'est un sujet de discussion, que ça soit sur le risque, ou sur l'économie. En France, non!

M. : Un des arguments des pronucléaires, est de dire qu'il n'y a pas d'alternative à l'arrêt des centrales, qu'arrêter le nucléaire c'est allumer des centrales à charbon, ce qui entraînerait de nombreux morts par la pollution de l'air.

B. L. : Mais personne n'a proposé de remplacer le nucléaire par des centrales à charbon. Ils comparent toujours ça. En Allemagne, ils ont fermé les centrales et ont diminué leur consommation de charbon. Pourquoi ? Parce que le renouvelable a plus que compensé l'apport du nucléaire. Même au Japon. J'ai fait une étude après Fukushima : l'arrêt de toutes les centrales nucléaires a eu pour effet une augmentation des émissions de gaz à effet de serre de $8 \%$, beaucoup moins que ce qu'ils prétendent, et si la facture énergétique du Japon a augmenté, c'est essentiellement parce que le prix mondial du pétrole a augmenté ces annéeslà et non à cause de l'arrêt du nucléaire. Comme le prix du pétrole est redescendu depuis, on se retrouve dans une situation de facture énergétique plus basse.

Personne n'a voulu remplacer le nucléaire par du charbon! À la limite, on peut faire le maximum de renouvelable et faire un peu de gaz à cycle combiné qui est beaucoup moins émetteur de $\mathrm{CO} 2$ que le charbon et a un bon rendement.

Personne ne nous a jamais dit que ce que l'on disait était faux. On nous dit que l'on a tort, c'est très différent. Global Chance est connu pour ça : on donne des chiffres. On a déjà fait une erreur, une seule en vintcinq ans, et on a reconnu notre erreur. On est reconnu comme disant des choses qui ne sont pas fausses. Mais eux, ils disent des choses fausses par ignorance, ou ils mentent, en permanence. Quant à la grande majorité des politiques, il est très rare qu'ils cherchent à savoir. Ils répètent.

La plupart des députés sont pronucléaires, mais ne savent pas comment fonctionne une centrale nucléaire ni ce qu'est le sodium. L'ENA est pronucléaire, pourquoi ? Parce que pour faire carrière en France il faut être pronucléaire. Les ambassadeurs ont comme premier travail de vendre des armes et du nucléaire, que ça soit au Bangladesh ou au Burkina. J'ai eu deux exemples sur la haute administration. Le premier est qu'à l'ENA, des étudiants avaient fait des groupes de travail sur l'efficacité énergétique, et ça a été très mal vu... Le second, j'ai rencontré en Chine un élève de l'ENA qui faisait un stage à l'ambassade de France et il m'a dit : " je suis surpris, je me pose des questions sur le nucléaire, mais toute l'ambassade est pronucléaire ". J'étais effrayé. Il n'y a aucune raison que l'ENA soit pronucléaire. Et les scientifiques critiques qui interviennent sur le nucléaire, au CNRS, il n'y en a pratiquement pas... 


\section{M. : L'État est hermétique à la critique du nucléaire?}

B. L. : L'expertise indépendante n'existe pratiquement pas, elle n'est que militante. Elle ne vient pas du monde universitaire ou des écoles d'ingénieurs. Elle vient des associations, c'est très bien, mais c'est minuscule. La CRIIRAD ${ }^{3}$, l'ACRO ${ }^{4}$, le GSIEN, Greenpeace, le Réseau Sortir du nucléaire et quelques autres associations antinucléaires font un bon travail, mais cela reste faible et n'est pas considéré du point de vue de la respectabilité, à tort à mon avis, comme un institut, une université ou un organisme scientifique reconnu.

On ne trouve pas d'institut public qui va faire une étude sérieuse. Dominique Voynet avait obtenu de Lionel Jospin, alors Premier ministre, une étude indépendante sur les coûts du nucléaire. Ce qui n'existait pas. Il n'y avait rien, sauf EDF qui disait ce qu'elle voulait. Il a fallu six mois pour trouver les trois personnes qui rédigeraient cette étude. Dominique Voynet avait demandé que Benjamin Dessus y participe. On lui a demandé de proposer d'autres noms, mais elle a tenu bon. À la fin, ils ont accepté Benjamin. Il y avait aussi l'administrateur général du CEA, pronucléaire par définition, et le commissaire au Plan, Michel Charpin, plutôt pronucléaire, mais jouant un peu le rôle d'arbitre. Ils ont travaillé pendant un an, avec des gens du CEA et d'autres organismes. Un travail remarquable et extrêmement fourni qui en particulier mettait en évidence que la filière MOX était beaucoup plus chère que toutes les autres solutions. Et tout ça signé par les trois. Le rapport, édité à la Documentation française a été remis à Lionel Jospin, mais il ne s'est rien passé. Même pas une réunion des conseillers des ministres pour voir ce qu'ils pouvaient tirer du rapport. Rien!

Une autre expérience, plus personnelle. Après Tchernobyl, il y avait encore un réacteur qui fonctionnait. L'Europe voulait qu'il soit arrêté. La France et l'Allemagne, à cette époque, disaient " il faut arrêter le réacteur, mais il faut le remplacer par un autre, car l'Ukraine a besoin d'énergie, et nous pourrions le réaliser ". Et ils voulaient que la Banque Européenne pour la Reconstruction et le Développement (BERD) finance le projet. Je n'étais pas encore au cabinet de Dominique Voynet. J'avais regardé ce problème en liaison avec la BERD pour montrer que l'Ukraine n'avait pas besoin de ce réacteur qui représentait $3 \%$ de la production d'électricité, et qu'elle avait des surcapacités de centrales thermiques fossiles. Qu'il valait mieux donner du gaz ou du charbon que lui faire payer une centrale. Arrive 1998, le gouvernement allemand décide la sortie du nucléaire et par conséquent de ne plus soutenir ce projet. Le gouvernement français, furibond, demande la création d'un groupe commun pour essayer d'obtenir une inflexion de cette décision et je participe à ce groupe de travail, côté français. Dominique Voynet en avait marre de ces histoires et me demande un rapport pour montrer que la position française n'est pas défendable. Je travaille mes week-ends dessus. Et j'explique la surcapacité, le ceci, le cela. Elle remet ce rapport au Premier ministre, en espérant qu'il y aurait une réunion pour dire : "j'ai posé un rapport, est-ce qu'il y a un rapport de l'autre côté qui justifie le projet? "Et il n'y a eu aucune
3. Commisson de Recherche et d'Information Indépendantes sur la Radioactivité.

4. Association pour le Contrôle de la Radioactivité dans l'Ouest. 
réponse, aucun rapport. La seule réaction a été de demander à ce que le rapport de Voynet reste confidentiel... Au final, la centrale n'a pas été faite, car les Allemands se sont retirés, et parce que la BERD a refusé le projet. Mais cette histoire montre comment cela fonctionne. Personne ne contre-argumente : rien! Personne n'a dit que le rapport Charpin-DessusPellat était faux. Personne n'a dit que mon rapport était faux. La seule réponse a été le silence. La "raison d'État " dit qu'il faut faire un réacteur en Ukraine, mais personne n'explique l'intérêt, sauf celui de rapporter des sous à EDF et Areva.

Alors ça marche un certain temps ce genre de stratégie, au niveau des grandes déclarations, mais dans la réalité ça ne fonctionne pas. En 1974, le grand argument pour faire avaler le projet de construction de six réacteurs par ans, c'était de dire que c'était six les premières années, puis quatre pour la France et deux vendus à l'étranger, puis deux pour la France et quatre pour l'étranger. Ça devait faire pas mal d'exportation sur quarante ans... mais on en a vendu huit en tout. Donc la stratégie s'est révélée fausse. Mais au lieu de la corriger, on continue de prétendre qu'on va vendre des EPR à l'Inde et partout. 